\title{
3D simulations of the multi-wavelength emission from young supernova remnants including efficient particle acceleration
}

\author{
Gilles Ferrand* \\ Dept. of Physics and Astronomy, University of Manitoba, Winnipeg MB R3T 2N2, Canada \\ E-mail: gferrandephysics.umanitoba.ca \\ Samar Safi-Harb ${ }^{\dagger}$ \\ Dept. of Physics and Astronomy, University of Manitoba, Winnipeg MB R3T 2N2, Canada \\ E-mail: samar.safi-harb@umanitoba.ca \\ Anne Decourchelle \\ Laboratoire AIM (CEA/Irfu, CNRS/INSU, Université Paris VII), CEA Saclay, bât. 709, 91191 \\ Gif sur Yvette, France \\ E-mail: anne.decourchelledcea.fr
}

\begin{abstract}
Within our Galaxy, supernova remnants (SNRs) are believed to be the major sources of cosmic rays, up to the knee of the cosmic ray spectrum. The detection of non-thermal radiation from SNRs, in X-rays over the past two decades, and finally in $\gamma$-rays over the past decade, has proved the presence of energetic particles in these objects. However important questions remain regarding the share of the hadronic and leptonic components, and the fraction of the supernova energy channelled into these. If protons are efficiently accelerated at the blast wave, this should impact the dynamics of the SNR itself, and therefore its morphology and spectral properties. In this paper we show how these effects can be investigated by the means of 3D numerical simulations. Our code couples the hydrodynamic evolution of the SNR with a non-linear kinetic model of diffusive shock acceleration, which allows us to produce synthetic projected maps to be compared with observations. We show how the presence of energetic protons can be inferred from the broadband emission of SNRs: 1) relativistic particles modify the properties of the shocked plasma, which affects the thermal X-ray emission, and 2) streaming particles can amplify the ambient magnetic field, which affects the non-thermal radio to $\gamma$-ray emission.
\end{abstract}

Cosmic Rays and the InterStellar Medium - CRISM 2014,

24-27 June 2014

Montpellier, France

\footnotetext{
* Speaker.

${ }^{\dagger}$ Canada Research Chair.
} 


\section{Context}

Based on energetic considerations, the most plausible sources of cosmic rays within the Galaxy have been identified as being supernova remnants (SNRs). And a theory has been developed, known as diffusive shock acceleration (DSA), which explains how charged particles swept up by a magnetized shock wave can get accelerated to very high energies [5, 12]. However it is still unclear which particles are accelerated inside SNRs, in which fractions, and to which energies.

Modern X-ray space observatories (such as Chandra, XMM-Newton, and Suzaku) allow to probe the structure of young remnants in great detail [14], and observations of objects like Tycho's SNR (G120.1+1.4) and SN 1006 (G327.6+14.6) have provided evidence that part of the kinetic energy of the explosion is channeled into energetic particles [11]. Also the cut-off of the synchrotron emission proves the acceleration of electrons to $\mathrm{TeV}$ energies. New $\gamma$-ray instruments in space (Fermi, Agile) and on the ground (H.E.S.S., VERITAS, MAGIC) have provided a new view of SNRs at the highest energies, but have not firmly identified Pevatrons yet. The difficulty here is to disentangle hadronic and leptonic contributions.

\section{Method}

We simulate the evolution of young SNRs undergoing particle acceleration as follows. Starting from self-similar profiles [4], the time-evolution of the shocked structure is computed using the 3D hydrodynamical code RAMSES [13], in a comoving grid of variable resolution [10]. The acceleration of particles at the forward shock is computed with a non-linear semi-analytical model [2], that provides the level of back-reaction on the fluid. Shock modifications are implemented in the hydro code through the use of a variable adiabatic index, which is lowered at the shock front according to the contribution of the particle pressure to the total pressure [6, 7]. The acceleration model includes recipes for the amplification of the magnetic field by the particles streaming in the precursor [3, 1$]$. At the end of the hydro simulation (of one octant of the SNR), each cell is processed to obtain the properties plasma and compute its X-ray thermal emission [8] and its broad-band non-thermal emission [9]. The emission is projected along the line of sight to generate synthetic maps and spectra.

The thermal emission, in the X-ray band, depends on the plasma density (obtained form the hydro code), the electron temperature (computed under the assumption of progressive equilibration with protons) and the ionization states of all species in the plasma (that has to be solved out of equilibrium). It is computed for 14 elements from Hydrogen to Nickel.

The non-thermal emission, in a broad band from radio to $\gamma$-rays, depends on the density (for the production and decay of pions by protons), on the magnetic field (for the synchrotron emission of electrons), and on the ambient photon fields (for the inverse Compton scattering by electrons). It is obtained after computing the evolution of particle spectra downstream of the forward shock, including adiabatic and radiative losses.

\section{Results}

We summarize here the most salient features from our simulated data, regarding the impact of accelerated particles on the SNR morphology and spectrum. 


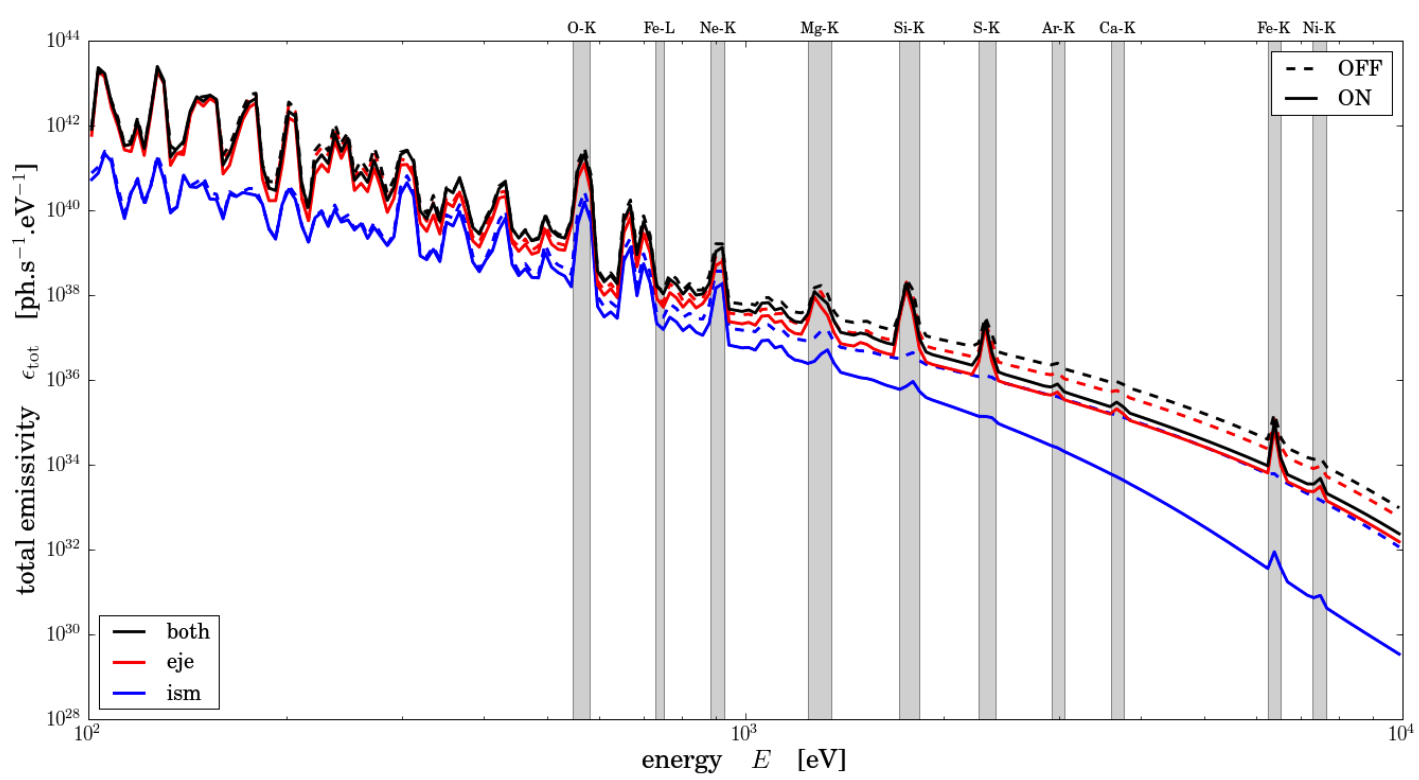

Figure 1: Thermal spectra (continuum + lines), spatially integrated over the the whole remnant (black), in the shocked ejecta (red), and in the shocked ISM (blue). Cases without (OFF, dashed) and with (ON, solid) the back-reaction of particles are compared. (Figure from [8])

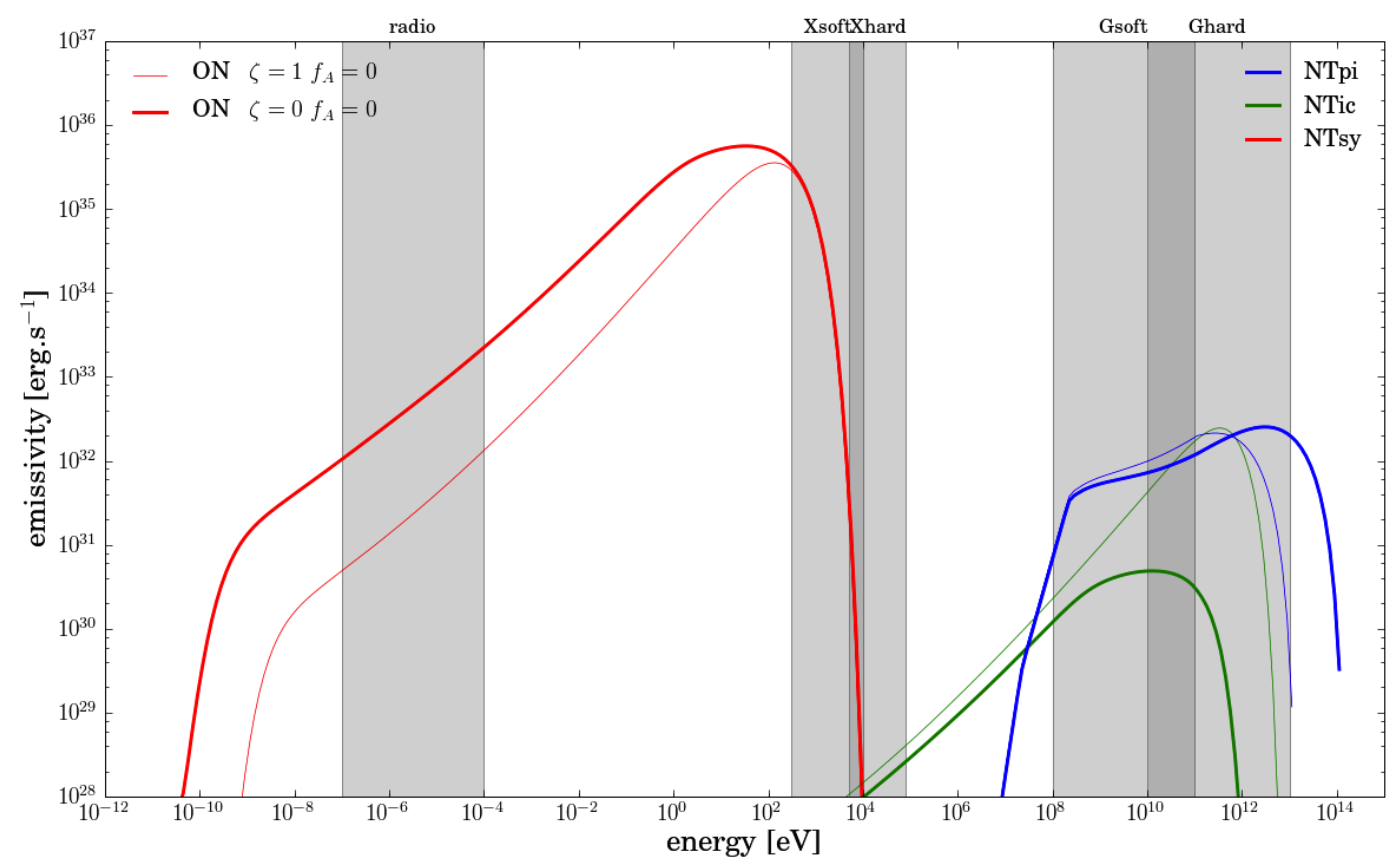

Figure 2: Non-thermal spectra from electrons (synchrotron in red and inverse Compton in green) and protons (pion decay in blue), integrated over the whole remnant. Cases without $(\zeta=1$, thin) and with $(\zeta=0$, solid) efficient magnetic field amplification are compared (here with back-reaction). (Figure from [9]) 


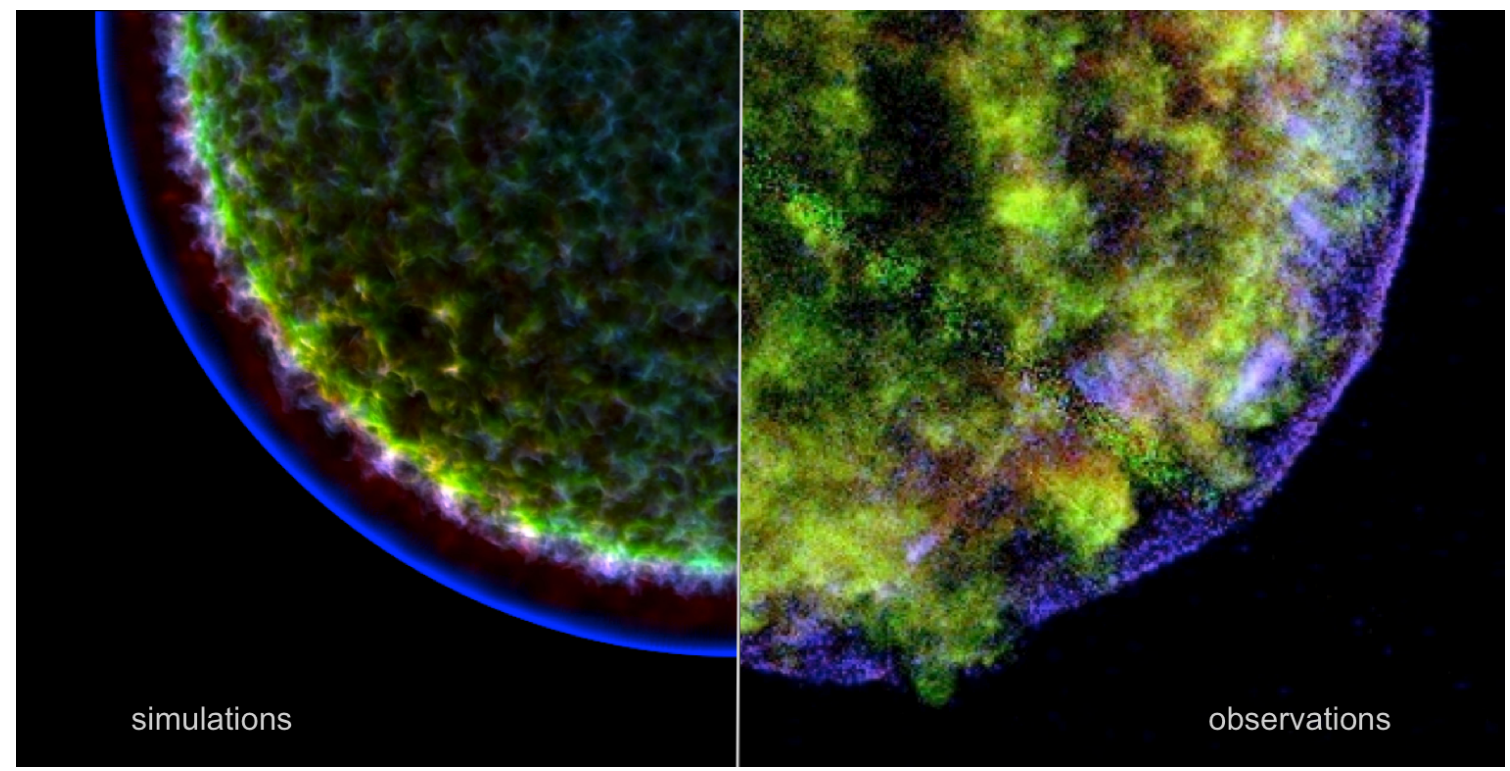

Figure 3: Left: X-ray image of a Tycho-like SNR at $t=500 \mathrm{yr}$, obtained by projection of a $1024^{3}$ simulation. Right: Part of a composite X-ray image of Tycho's SNR made from Chandra data [15]. Both images combine thermal emission from the hot ejecta (the irregular structures in the centre) and non-thermal emission from the energetic electrons (the outer rim). (Note that the comparison is work in progress, the colours of the ejecta in the simulated image do not match those in the observed image at this point.)

Figure 1 shows integrated thermal spectra in X-rays, in the ejecta and the ISM, with or without taking into account the back-reaction exerted by the particles accelerated at the shock. When particles are efficiently accelerated, we observe that the emission from the plasma is lowered, all the more so for higher photon energies. We have shown how observing the SNR in different energy bands, corresponding to different lines of different elements, allows to probe different levels of the impact of particle back-reaction [8].

Figure 2 shows integrated non-thermal spectra from the radio to $\gamma$-rays, in the case with efficient particle back-reaction, with or without efficient amplification of the magnetic field by the particles upstream of the shock. We observe that high magnetic fields $(>100 \mu \mathrm{G})$, as observed in young SNRs [14, and references therein], directly impact the synchrotron emission from electrons, by restricting their emission to thin rims, and indirectly impact the inverse Compton emission from electrons and also the pion decay emission from protons, mostly by shifting their cut-off energies to respectively lower and higher energies [9].

Figure 3 shows an illustrative side-by-side comparison of our simulations with observations of Tycho's SNR, in the X-ray domain, with both thermal and non-thermal emissions. Although this is not an actual fit of the data, it shows the kind of detailed morphological features that can be produced with our model.

\section{Perspectives}

Simulations were made so far for an idealized case appropriate for a type Ia supernova, the next step is to study the impact of the supernova explosion and of the environment where it occurs. We 
will use more realistic profiles for the ejecta of various supernova types, bridging the gap between studies of SNRs and the SNe.

We will also study the role of the progenitor star, by considering the propagation of a blast wave in the wind of a massive star. This will be relevant to core-collapse SNRs (types Ib/c and II). We are also interested in the interaction of the shell and the particles with a molecular cloud, a situation that enhances hadronic emissions (and will require the addition of radiative cooling).

We have considered here acceleration at the forward shock only, we may investigate the role of the reverse shock as well. We could also improve on the treatment of the contact discontinuity between the ejecta and the ISM, by using the MHD version of RAMSES, to investigate how the magnetic field affects the development of the Rayleigh-Taylor instability.

\section{Acknowledgments}

This research was primarily supported by NSERC through the Canada Research Chairs Program, and in part by the Canadian Institute for Theoretical Astrophysics through the National Fellows Program. It was also partially funded by the ACCELRSN project ANR-07-JCJC-0008 and by the COSMIS project ANR-10-BLAN-0509 in France.

The numerical simulations were performed: on the Titane super-computer at CEA/CCRT (France); on the Nuit computing cluster at the University of Manitoba, funded by Canada's Foundation for Innovation and the Manitoba Research and Innovation Funds program; and on the Grex, Orcinus and Parallel super-computers of the WestGrid consortium, the regional division of Compute Canada.

\section{References}

[1] A. R. Bell, Cosmic ray acceleration, Astroparticle Physics 43 56-70, 2013

[2] P. Blasi, S. Gabici, and G. Vannoni, On the role of injection in kinetic approaches to non-linear particle acceleration at non-relativistic shock waves, MNRAS 361 907-918, 2005 [astro-ph/0505351]

[3] D. Caprioli, et al, Dynamical effects of self-generated magnetic fields in cosmic-ray-modified shocks, ApJ 679 L139-L142, 2008 [astro-ph/ 0804.2884 ]

[4] R. A. Chevalier, Blast waves with cosmic-ray pressure, ApJ 272 765-772, 1983

[5] L. O. Drury, An introduction to the theory of diffusive shock acceleration of energetic particles in tenuous plasmas, Reports on Progress in Physics 46 (8) 973-1027, 1983

[6] D. C. Ellison, et al, Particle acceleration in supernova remnants and the production of thermal and nonthermal radiation, ApJ 661 879-891, 2007 [astro-ph/0702674]

[7] G. Ferrand, et al, 3D simulations of supernova remnants evolution including non-linear particle acceleration, A\&A 509 L10, 2010 [astro-ph/0912 .4886]

[8] G. Ferrand, A. Decourchelle, and S. Safi-Harb, Three-dimensional simulations of the thermal X-ray emission from young supernova remnants including efficient particle acceleration, ApJ 760 34, 2012 [astro-ph/1210.0085] 
[9] G. Ferrand, A. Decourchelle, and S. Safi-Harb, Three-dimensional simulations of the non-thermal broadband emission from young supernova remnants including particle acceleration, ApJ 78949 2014 [astro-ph/1405.0614]

[10] F. Fraschetti, et al, Simulation of the growth of the 3D Rayleigh-Taylor instability in supernova remnants using an expanding reference frame, A\&A 515 A104, 2010 [astro-ph/1002.5048]

[11] E. A. Helder, et al, Observational signatures of particle acceleration in supernova remnants, Space Sci. Rev. 173 369-431, 2012 [astro-ph/1206.1593]

[12] M. A. Malkov and L. O. Drury, Nonlinear theory of diffusive acceleration of particles by shock waves, Reports on Progress in Physics 64 (4) 429-481, 2001

[13] R. Teyssier, Cosmological hydrodynamics with adaptive mesh refinement. A new high resolution code called RAMSES, A\&A 385 337-364, 2002 [astro-ph/0111367]

[14] J. Vink, Supernova remnants: the X-ray perspective, A\&A Rev. 20 49, 2012 [astro-ph/1112.0576]

[15] J. S. Warren, et al., Cosmic-Ray Acceleration at the Forward Shock in Tycho's Supernova Remnant: Evidence from Chandra X-Ray Observations, ApJ 634 376-389, 2005 [astro-ph/ 0507478 ] 